\title{
Polymethylmethacrylate (PMMA) Augmentation Of A Cannulated And Fenestrated Pedicle Screw Fixation For Stabilization Of The Lumbar Degenerative Disease Accompanied With Osteoporosis
}

\author{
Mohd mohasin khan ${ }^{1}$ Mohammad sahamim ${ }^{2}$ Cui xue wen ${ }^{3}$ et al. \\ ${ }^{1,2,3}$ Department of Orthopedics,Affiliated Hospital of Jiangsu university, ZhenJiang 212001, China \\ Corresponding email: Cui5898@163.com
}

\begin{abstract}
:
Objective: To explore the clinical efficacy of the use of polymethylmethacrylate (PMMA) augmentation of a cannulated and fenestrated pedicle screwfixation for the treatment of lumbar degenerative disease accompanied withosteoporosis.

Methods: Thirty-one patients of lumbar degenerative disease accompanied with osteoporosisfrom Jun 2008 to Jan 2013 were selected .They were 11 males and 20 females withaverage age of 73. 5 years including lumbar degenerative stenosis 14 cases, lumbar intervertebral discherniation combined with segmental instability 9 cases, lumbar degenerative spondylolisthesis 6 cases, andlumbar degenerative scoliosis 2 cases. The patients were treated with lumbar canal decompression, fusion and polymethylmethacrylate (PMMA) augmentation of a cannulated and fenestrated pedicle screw fixationaccording to their clinicalfeature and imaging.

Results: All cases were followed up for 40 months (range,36-48months). No screw breakage, rod breakage, screw extraction, loosening, pseudoarticulation formation, or incision infection wasfound.The postoperative VAS score wasremarkably lower than preoperative score $(P<0.05)$. The JOAscore were improved significantly $(P<0.05)$.

Conclusion: For patients suffering from lumbar degenerative disease accompanied with osteoporosis, PMMA augmentation of a cannulated and fenestrated pedicle screwfixation was helpful for increasing the stabilization of screw and preventing from loosen and dislocation of the screws, therefore beneficial for improvement and maintenance of clinical efficacy.
\end{abstract}

Key word: Osteoporosis; cannulated and fenestrated pedicle screw; PMMA; Lumbar degenerative disease

\section{Introduction}

Lumbar degenerative disease is common in the elderly people, patients often associated with severe degenerative lumbar spinal stenosis, degenerative lumbar instability and scoliosis, causing the corresponding clinical symptoms, which are mainly surgical spinal decompression therapy, some patients need to pedicle screw fixation in the lumbar spine to maintain stability and promote fusion. Due to varying degrees of osteoporosis in many elderly people, vertebral bone loss due to this internal fixation cannot provide a solid anchor point or bone support, with a fixed load into the interface can cause damage arising from late loosening and falling, resulting in failure of internal fixation. So be firm pedicle screw in osteoporosis patients is very difficult [1]. In order to improve osteoporosis pedicle screw fixation strength, we used poly (methyl methacrylate) (Polymethylmethacrylate, PMMA) bone cement augmentation of pedicle screws to the side hole of the hollow increases in osteoporotic vertebral pedicle screw the gripping force [2 ]. Experiments show that this new type of pedicle screws has a stronger biomechanical property [3]. For such patients we first application of bone cement reinforced hollow side hole fixation of pedicle screws, and then the corresponding segments of the spinal canal decompression, or post- surgical lateral interbody fusion, and achieved good results.

\section{Materials and methods:}

2.1 General study of 31 cases group, DEXA bone density test instrument for the diagnosis of osteoporosis. Including 11 males and 20 females, aged of 65 to 86 years, mean 73.5 years old; All patients were with varying degrees of back pain to stand, walk, postural changes significantly, slightly alleviated when supine rest, nonsteroidal anti-inflammatory drug therapy had no significant effect. 17 patients with lower limb exists radiating pain, numbness, weakness and other radicular symptoms, 22 cases had existence of intermittent claudication symptoms, in such types of cases non-surgical treatment are ineffective. Preoperative lateral lumbar flexion and extension was checked by X-ray film and digital power lumbar spine CT, MRI examination. Clinical diagnosis: 14 cases of lumbar spinal stenosis and lumbar disc herniation instability in nine cases, six cases of degenerative 
lumbar spondylolisthesis, degenerative lumbar scoliosis in two cases. 16 cases of diabetes, hypertension in 14 cases, 3 cases of coronary heart disease, preoperative medical treatment after exclusion surgery taboo.

2.2 Procedures were based on clinical and imaging data,Determine decompression method, fusion segments.Endotracheal intubation under general anesthesia, prone position, after incision approach, segmental lesions revealed bilateral lamina and pedicle screw entry point, opening the cone opening, at a certain angle and direction, along the vertebral pedicle the guide into $\varphi 1 \mathrm{~mm}$ Kirschner, C-arm X-ray fluoroscopy to determine the correct fixed segment, Kirschner good location, select the appropriate length and diameter of the hollow side holes pedicle screws screwed into the vertebral body along the guide Kirschner the length of pedicle screws to the front tip of the vertebral body $1 / 3$ is appropriate, in the C-arm X-ray machine to monitor the pressure will slowly inject the bone cement hollow pedicle screws, pedicle screws from bone cement tip and side holes overflow to the vertebral dispersed to avoid leakage of bone cement into the spinal canal and paraspinal average on each side of the pedicle inject about $2.0 \mathrm{ml}$. Then reduced lesion segments of integration.Adopt the characteristics of the lesion fenestration, semi laminectomy or laminectomy and lateral recess augmentation, the full release of nerve root.Exploration disc, there are prominent portions to be removed. After the completion of decompression has been completely solidified cement, placed fixed and orthopedic fixation rods, pre-bent rods through a modest recovery in lumbar lordosis and scoliosis, reduced bone tissue resected bone cut grain, or the side of the rear row intervertebral fusion.

3.3 After surgical treatment prophylactic use of antibiotics, postoperative hormonal, neural nutrition and dehydration agent are given as symptomatic and supportive treatment. $48 \sim 72 \mathrm{~h}$ after pulling drainage tube, the first three days from wearing lumbosacral brace ambulation, assisted by anti-osteoporosis drug therapy, brace equipped with 3 to 6 months. Lumbar lateral X-ray film is taken at one week, three months, six months later, to observe the situation and internal fixation and fusion loose or unplugged.

2.4 Evaluation of the patient clinical condition is done by the VAS pain score before and after surgery.

0 painless, 10 into pain, $<3$ into a good 3 to 4 divided basically satisfied, $\geq 5$ into poor. Neurological symptoms using the Japanese Orthopaedic Association (JOA) 29 of Law on neurological and life skills before surgery and follow-up of the score, the improvement rate is calculated: improvement rate $=$ (follow-up JOA score preoperative JOA score) / (29 - surgery former JOA score $) \times 100 \%$ were evaluated.Radiographic assessment of fusion reference Sengupta et al [4] Standard: According to X-ray films will be integrated into three conditions: (1) strong bony fusion, that there is continuity of bone callus formation zone, fixed fusion segments no unusual activity; (2) inaccurate fusion, fusion does not meet the strong nor pseudarthrosis; (3) pseudarthrosis, that the district had no significant bone callus formation, pedicle screws are loose, Section activity of more than $5^{\circ}$ segments 1.5 Statistical analysis Statistical analysis was performed using SPSS 13. 0 software for data processing. Clinical efficacy using two-sample t-test statistics, $\mathrm{P}<0.05$ were considered statistically significant.

\section{Result:}

31 patients underwent follow-up of 36 to 48 months (mean 40 months). The group of 23 cases of secondary complications, including 5 cases of bone cement into patients undergoing lumbar paraspinal muscle gap, 3 cases of vertebral venous infiltration signs, two cases of bone cement leakage to the upper and lower vertebral space. Due to the small amount of leakage, nerve and blood vessel damage and other serious complications of pulmonary embolism occurred; intraoperative dural tear in 2 cases, postoperative cerebrospinal fluid leakage, prolonged drainage time to one week, by changing dressings, wound pressure and prone position and other measures taken to heal. 4 cases of delayed wound healing; 3 cases of postoperative pulmonary infection symptoms, 2 patients had digestive disorders, 2 cases of deep vein thrombosis, or have medical treatment after symptomatic improvement. No perioperative deaths.

Preoperative and postoperative neurological symptoms last follow-up mitigation JOA score, back pain and leg pain VAS score results are shown in Table 1 . After surgery neurological symptoms mitigation JOA $(\mathrm{P}<0$. 01) comprehensive back pain, leg pain in the patients were assessed individually and it was significant improvement $(\mathrm{P}<0.01)$. Evaluation criteria based on $\mathrm{X}$-ray films and 22 patients achieved a strong bone fusion, nine cases are imprecise fusion, does not appear broken nails, broken rods, pedicle screws loose or prolapse, fusion segments pseudarthrosis formation. 
Polymethylmethacrylate (PMMA) Augmentation Of A Cannulated And Fenestrated Pedicle ...

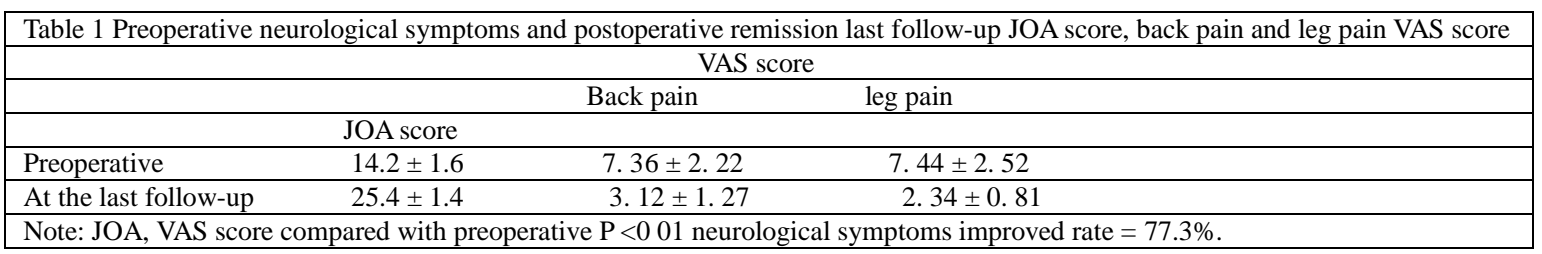

\section{Discussion:}

Mainly for degenerative lumbar degenerative disc bulging or protruding, facet hypertrophy, yellow ligament hypertrophy, segmental instability, scoliosis can also occur, such as changing or rotating, the major clinical manifestations of lumbar spinal stenosis, disc herniation, vertebral instability or scoliosis, etc., associated with osteoporosis will increase above degeneration. Severe symptoms often require surgical treatment, surgery is decompression and fixation, the majority of elderly patients due to the inherent instability and lumbar scoliosis, etc., and the pressure will increase the progress of surgery widespread instability and lumbar scoliosis, so most fixed stabilize the spine and orthopedic surgery needs within the pedicle screw system.

For patients with osteoporosis, bone loss often occur pedicle screw loose, pull, integration failed pseudarthrosis and other complications. Studies have reported reduced bone mineral density will affect the maximum axial pedicle screw pull-out strength, so patients with severe osteoporosis is often considered the pedicle screw fixation surgery contraindications. Therefore, to improve osteoporosis bone - screw interface is the key to solving these problems.Bone - screw interface stability in general and BMD, screw geometry, screw fixation technologies. There are many methods currently used clinically to improve the stability of the pedicle screw, the side hole of the hollow bone cement augmentation of pedicle screw is a reliable method of [5], so that after the bone cement augmentation of pedicle screw - the bone-cement interface and bone cement - bone interface connection strength greatly enhanced bone cement in vertebral cancellous bone diffusion, increase the contact surface with the bone, the screw has been enhanced stability [6,7]. Hollow side pedicle screw holes in recent years developed a new type of pedicle screws, and its design features are hollow screw tip with side holes, making the bone cement through this pathway diffuse into the surrounding bone screws through screws and bone cement anchors, bone cement and bone surrounding the screw anchors fixed to enhance capacity. Therefore, the authors used cement -reinforced hollow side to enhance the stability of the pedicle screw holes and screw holding power. As good fixation stability, severe lumbar instability, severe spinal stenosis, may also laminectomy and facet cut, completely release the nerve root, to ensure the outcome. In addition, the cytotoxic effect of bone cement polymethyl methacrylate monomer structural damage sensitive nerve endings and bone cement during polymerization heat released causes pain nerve damage can play a good analgesic effect, which patients pain relief plays an important role. Polymethyl methacrylate bone cement its complications is the main problem arising from the presence of the surgical procedure, heat polymethyl methacrylate bone cement with damaging effects of the heat of polymerization can be produced by the surrounding bone tissue damage, misuse bone cement injection and dangerous spill damage the spinal cord and nerve roots. In order to facilitate the operation of surgery, using a 1:1 configuration viscosity bone cement, which requires the injection of bone cement injection timing and quantity of strict grasp, too thin or too early volume more easily penetrate the blood vessels and the spinal canal, too late too thick or less, reducing trabecular bone cement squeeze, would not achieve enhanced results. Therefore, the injection process must be continued under fluoroscopy slow bone cement is injected into the vertebral body compression, bone cement under fluoroscopic monitoring the amount and injection rate. Author's experience is that unilateral pedicle injection volume to about $2 \mathrm{~mL}$ appropriate. This group of patients after the first $3 \sim 5 \mathrm{~d}$ to get out of bed without feeling abnormal, indicating the efficacy of internal fixation played a supporting role. Bone cement augmentation of pedicle screw hole hollow side is relatively complicated surgical procedure that prolonged operative time, increased bleeding, trauma increases; increase the economic costs, increased surgical complications. Early in this group of patients, 23 cases of postoperative complications times, although not serious dysfunction, but the tips that we should strictly control the fixed indications, as far as possible within the limited use of fixed, fixed within the control segment and the number. Intraoperative caution to prevent leakage of bone cement damage nerve roots.

Intraoperative fluoroscopy must be confirmed before the injection of bone cement pedicle screw entirely within the pedicle and vertebral body, due to the vertebral venous drainage $1 / 3$ is located in the posterior pedicle screw tip should be located in front of the vertebral body $1 / 3$. The timing and amount of bone cement injected strict grasp, too thin or prone to excessive leakage of bone cement, and then injected into difficulties too thick, less then reach strengthening effect. The patients on average each side of the pedicle injected about $2.0 \mathrm{ml}$, good intraoperative pedicle screw stability, pulling the reset and the process does not appear orthopedic screws loose or out phenomenon. 


\section{Conclusion:}

The use of bone cement reinforced hollow side holes spinal pedicle screw system, not only to provide immediate spinal stability, but also in patients with osteoporosis get lasting and strong fixation of the spine, help to improve the clinical efficacy and maintaining.

\section{Reference}

[1]. Ponnusamy, K E, et al. Instrumentation of the osteoporotic spine: biomechanical and clinical considerations. The Spine Journal, 2011. 11(1): p. 54-63.

[2]. Amendola, L, et al. Fenestrated pedicle screws for cement-augmented purchase in patients with bone softening: a review of 21 cases. Journal of Orthopaedics and Traumatology, 2011. 12(4): p. 193-199.

[3]. Zhuang, X M, et al. Effect of the degree of osteoporosis on the biomechanical anchoring strength of the sacral pedicle screws: an in vitro comparison between unaugmented bicortical screws and polymethylmethacrylate augmented unicortical screws. Spine, 2010. 35(19): p. E925-E931.

[4]. Sengupta DK, Truumees E, Patel CK, et al. Outcome of Local Bone Versus Autogenous Iliac Crest Bone Graft in the Instrumented Posterolateral Fusion of the Lumbar Spine. Spine, 2006, 31(9):985- 991.

[5]. Amendola, L, et al, Fenestrated pedicle screws for cement-augmented purchase in patients with bone softening: a review of 21 cases. Journal of Orthopaedics and Traumatology, 2011. 12(4): p. 193-199.

[6]. Takigawa T,Tanaka M, Konishi H,et al. Comparative biomechanical analysis of an improved novel pedicle screw withsheath and bone cement. J Spinal Disord Tech, 2007, 20(6):462- 467.

[7]. Tan JS, Bailey CS, Dvorak MF, et al. Cement augmentation ofvertebral screws enhances the interface strength between interbodydevice and vertebral body. Spine,2007, 32(3):334- 341.

\section{Images}

\section{Preoperative}
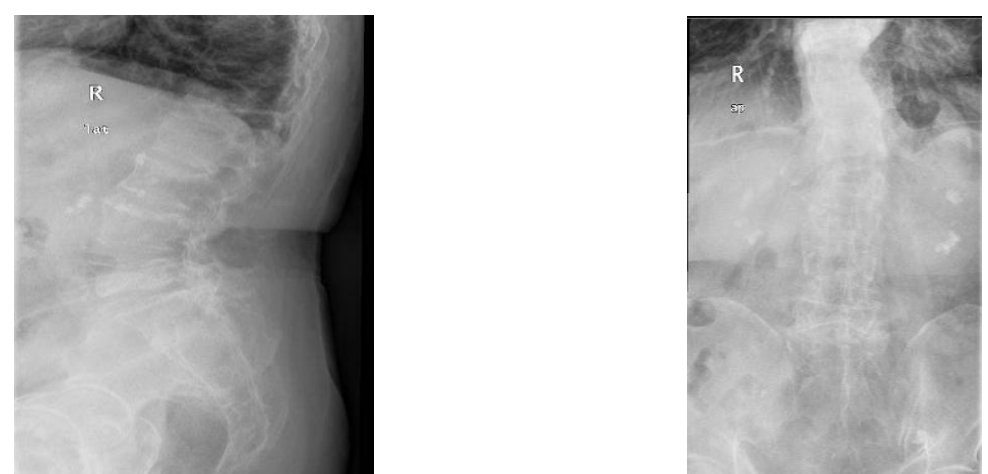

Figure 1 female patients, aged 78, leg pain, intermittent claudication, more than five years, preoperative lateral X-ray film showing lumbar degenerative disease, T12 (old, L2 (fresh) compression fractures, DEXA bone densitometer diagnosis of osteoporosis test
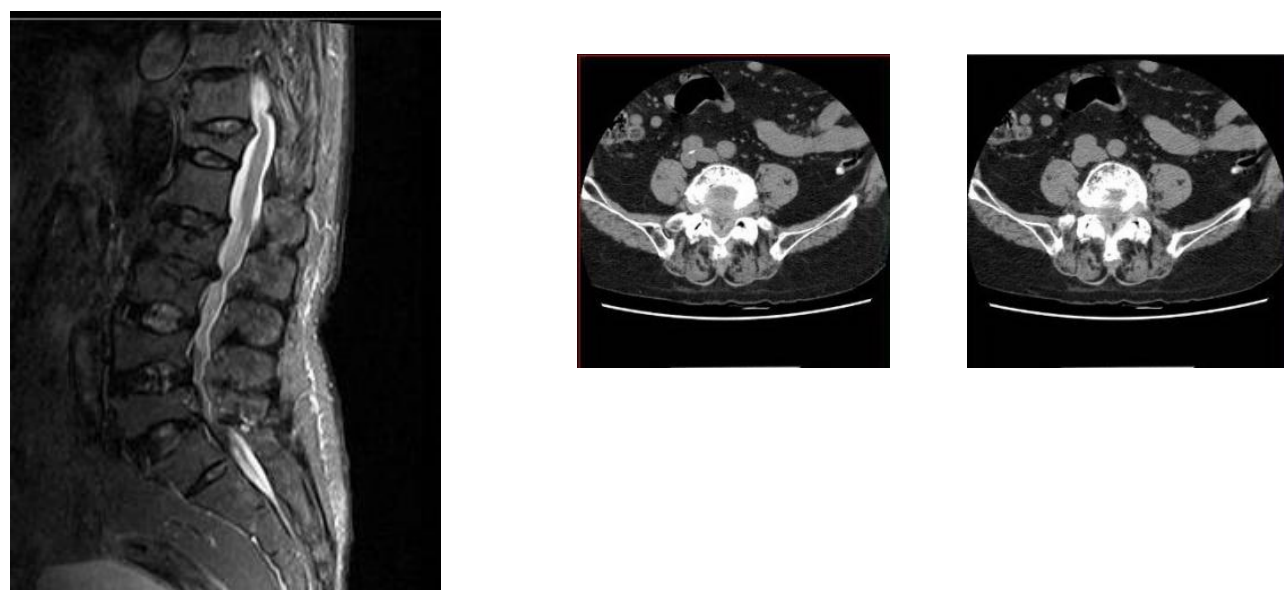

figure 2 Sagittal MRI film shows L2 / 3 disc, L segment S1 spinal stenosis; L4 / 5, L5 / S1 cross section showing severe spinal stenosis, yellow ligament hypertrophy, lateral recess stenosis 


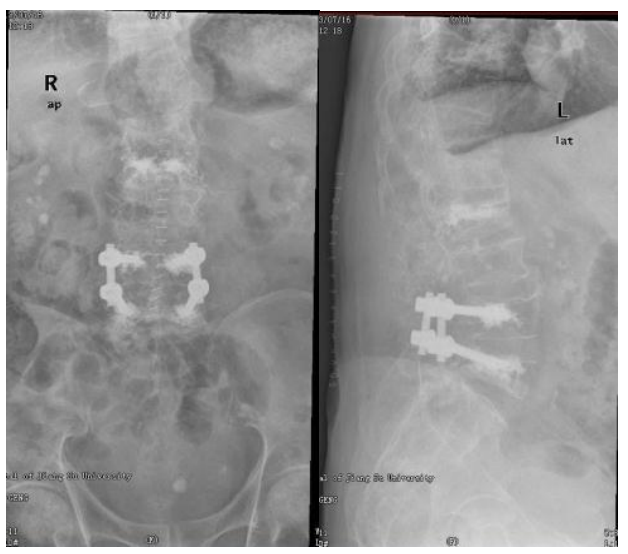

Figure 3 Postoperative lateral X-ray films, L4, 5 bone cement reinforced hollow side holes pedicle screw fixation is limited, good screw position, lumbar curvature of a good recovery, and no loose, pull out 